\title{
STRONG EQUIVALENCES OF APPROXIMATION NUMBERS AND TRACTABILITY OF WEIGHTED ANISOTROPIC SOBOLEV EMBEDDINGS
}

\author{
JIDONG HAO, HEPING WANG
}

\begin{abstract}
In this paper, we study multivariate approximation defined over weighted anisotropic Sobolev spaces which depend on two sequences $\mathbf{a}=$ $\left\{\mathbf{a}_{j}\right\}_{j \geq 1}$ and $\mathbf{b}=\left\{\mathbf{b}_{j}\right\}_{j \geq 1}$ of positive numbers. We obtain strong equivalences of the approximation numbers, and necessary and sufficient conditions on $\mathbf{a}, \mathbf{b}$ to achieve various notions of tractability of the weighted anisotropic Sobolev embeddings.
\end{abstract}

\section{INTRODUCTION}

This paper is devoted to investigating sharp constants of approximation numbers and tractability of embeddings of weighted anisotropic Sobolev spaces on $[0,1]^{d}$ into $L_{2}\left([0,1]^{d}\right)$. The approximation numbers of a bounded linear operator $T: X \rightarrow Y$ between two Banach spaces are defined as

$$
\begin{aligned}
a_{n}(T: X \rightarrow Y): & =\inf _{\operatorname{rank} A<n} \sup _{\|x\|_{X} \leq 1}\|T x-A x\|_{Y} \\
& =\inf _{\operatorname{rank} A<n}\|T-A\|_{X \rightarrow Y}, \quad n \in \mathbb{N} .
\end{aligned}
$$

They describe the best approximation of $T$ by finite rank operators. If $X$ and $Y$ are Hilbert spaces and $T$ is compact, then $a_{n}(T)$ is the $n$-th singular number of $T$. Also $a_{n}(T)$ is just the $(n-1)$-th minimal worst-case error with respect to arbitrary algorithms and general linear information in the Hilbert setting.

Recently, Kühn and many other authors investigated and obtained strong equivalences, preasymptotics, asymptotics of the approximation numbers and tractability of the classical isotropic Sobolev embeddings, Sobolev embeddings of dominating mixed smoothness, Gevrey space embeddings, anisotropic Sobolev embeddings on the torus $\mathbb{T}^{d}=[0,2 \pi]^{d}$ (see [11, 12, 10, 1]), and Sobolev embeddings and Gevrey type embeddings on the sphere $\mathbb{S}^{d}$ and on the ball $\mathbb{B}^{d}$ (see [2]). In [24] Werschulz and Woźniakowski investigated tractability of weighted isotropic Sobolev embeddings.

We note that unlike on the torus $\mathbb{T}^{d}=[0,2 \pi]^{d}$, the anisotropic spaces $W_{2}^{\mathbf{b}}\left([0,1]^{d}\right)$ on the torus $[0,1]^{d}$ naturally induce weighted norms given in terms of Fourier coefficients (see (2.4) in Section 2.1), where $\mathbf{b}=\left\{b_{j}\right\}_{j \geq 1}$ is a positive sequence describing smoothness index of each variable. In this paper we consider general weighted anisotropic Sobolev spaces $W_{2}^{\mathbf{a}, \mathbf{b}}\left([0,1]^{d}\right)$ on the torus $[0,1]^{d}$ whose definitions are

2010 Mathematics Subject Classification. 41A25, 41A63, 65D15, 65Y20.

Key words and phrases. Strong equivalences, Tractability, Approximation numbers, Weighted anisotropic spaces, Analytic Korobov space. 
given in Section 2.1, where the positive sequence $\mathbf{a}=\left\{a_{j}\right\}_{j \geq 1}$ is a scaling parameter sequence, and $\mathbf{b}=\left\{b_{j}\right\}_{j \geq 1}$ is a smoothness parameter sequence. We discuss the approximation numbers and tractability of the weighted anisotropic Sobolev embedding

$$
I_{d}: W_{2}^{\mathbf{a}, \mathbf{b}}\left([0,1]^{d}\right) \longrightarrow L_{2}\left([0,1]^{d}\right),
$$

where $I_{d}$ is the identity (embedding) operator.

We obtain strong equivalences of the approximation numbers $a_{n}\left(I_{d}\right) \equiv a_{n}\left(I_{d}\right.$ : $\left.W_{2}^{\mathbf{a}, \mathbf{b}}\left([0,1]^{d}\right) \rightarrow L_{2}\left([0,1]^{d}\right)\right)$ as $n \rightarrow \infty$. We remark that the sharp orders of $a_{n}\left(I_{d}\right)$ depend only on the smoothness parameter sequence $\mathbf{b}$, and the sharp constants are closely related to the volume of the generalized ellipsoid defined by $\mathbf{a}, \mathbf{b}$ and $d$. Our result generalizes Theorem 2.4 in [1]. However, we do not obtain results about preasymptotics of $a_{n}\left(I_{d}\right)$ as in 1 .

We also consider tractability of the approximation problem $I=\left\{I_{d}\right\}$ of the weighted anisotropic Sobolev embeddings. We consider algorithms that use finitely many continuous linear functionals. The information complexity $n\left(\varepsilon, I_{d}\right)$ is defined to be the minimal number of linear functionals which are needed to find an approximation to within an error threshold $\varepsilon$. There are two kinds of tractability based on polynomial convergence and exponential convergence. The classical tractability describes how the information complexity behaves as a function of $d$ and $\varepsilon^{-1}$, while the exponential convergence-tractability (EC-tractability) does as one of $d$ and $\left(1+\ln \varepsilon^{-1}\right)$. Nowadays the study of tractability and EC-tractability has attracted much interest, and a great number of interesting results have been obtained (see [14, 15, 16, 5, 6, 17, 21, and the references therein).

Denote by $H\left(K_{d, \mathbf{a}, 2 \mathbf{b}}\right)$ the analytic Korobov space which is a reproducing kernel Hilbert space with the reproducing kernel $K_{d, \mathbf{a}, 2 \mathbf{b}}$, and whose definition will be given in Section 2.2. Such space $H\left(K_{d, \mathbf{a}, 2 \mathbf{b}}\right)$ has been widely investigated in the study of tractability and EC-tractability (see [4, 5, 6, 7, 8, 13, 22]). Particularly, the papers [4, 22] considered different notions of EC-tractability of the approximation problems $\mathrm{APP}=\left\{\mathrm{APP}_{d}\right\}_{d \in \mathbb{N}}$, and obtained the corresponding necessary and sufficient conditions, where

$$
\mathrm{APP}_{d}: H\left(K_{d, \mathbf{a}, 2 \mathbf{b}}\right) \longrightarrow L_{2}\left([0,1]^{d}\right), \text { with } \mathrm{APP}_{d}(f)=f
$$

In this paper, we establish the relationship of the information complexities $n\left(\varepsilon, I_{d}\right)$ and $n\left(\varepsilon, \mathrm{APP}_{d}\right)$. Based on this relationship, we obtain necessary and sufficient conditions for various notions of tractability of the approximation problem $I=\left\{I_{d}\right\}_{d \in \mathbb{N}}$.

The paper is organized as follows. In Section 2 we introduce the weighted anisotropic Sobolev spaces, the analytic Korobov spaces, properties of the approximation numbers, tractability, and then state out main results. Section 3 is devoted to proving strong equivalence of the approximation numbers of the weighted anisotropic embeddings. In Section 4 we prove tractability of the weighted anisotropic embeddings.

\section{Preliminaries AND MAin Results}

\subsection{Weighted anisotropic Sobolev spaces on $[0,1]^{d}$.}


Denote by $L_{2}\left([0,1]^{d}\right)$ the collection of measurable functions $f$ on $[0,1]^{d}$ with finite norm

$$
\|f\|_{2}=\left(\int_{[0,1]^{d}}|f(\mathbf{x})|^{2} d \mathbf{x}\right)^{\frac{1}{2}}<+\infty .
$$

It is well known that any $f \in L_{2}\left([0,1]^{d}\right)$ can be expressed by its Fourier series

$$
f(\mathbf{x})=\sum_{\mathbf{k} \in \mathbb{Z}^{d}} \hat{f}(\mathbf{k}) e^{2 \pi i \mathbf{k x}}, \quad \mathbf{x} \in[0,1]^{d}
$$

where $i=\sqrt{-1}, \mathbf{k x}=\sum_{j=1}^{d} k_{j} x_{j}$, and

$$
\hat{f}(\mathbf{k})=\int_{[0,1]^{d}} f(\mathbf{x}) e^{-2 \pi i \mathbf{k x}} d \mathbf{x}, \quad \mathbf{k} \in \mathbb{Z}^{d},
$$

are the Fourier coefficients of the function $f$. We have the following Parseval equality:

$$
\|f\|_{2}=\left(\sum_{\mathbf{k} \in \mathbb{Z}^{d}}|\hat{f}(\mathbf{k})|^{2}\right)^{1 / 2} .
$$

For $r>0$, denote by $D_{j}^{r} f \equiv \frac{\partial^{r}}{\partial x_{j}^{r}} f$ the $r$-order partial derivative of $f$ with respect to $x_{j}$ in the sense of Weyl, i.e.,

$$
D_{j}^{r} f(\mathbf{x})=\sum_{\mathbf{k} \in \mathbb{Z}^{d}}\left(2 \pi i k_{j}\right)^{r} \hat{f}(\mathbf{k}) e^{2 \pi i \mathbf{k x}}, \quad\left(2 \pi i k_{j}\right)^{r}=\left|2 \pi k_{j}\right|^{r} \exp \left(\frac{r \pi i}{2} \operatorname{sign} k_{j}\right) .
$$

It follows from the Parseval equality that for $D_{j}^{r} f \in L_{2}\left([0,1]^{d}\right)$,

$$
\left\|D_{j}^{r} f\right\|_{2}=\left(\sum_{\mathbf{k} \in \mathbb{Z}^{d}}\left|2 \pi k_{j}\right|^{2 r}|\hat{f}(\mathbf{k})|^{2}\right)^{1 / 2} .
$$

Now we define weighted anisotropic Sobolev spaces. Let $\mathbf{a}=\left\{a_{k}\right\}_{k \geq 1}$ and $\mathbf{b}=\left\{b_{k}\right\}_{k \geq 1}$ be two sequences of positive numbers. Usually, we assume that the sequences $\mathbf{a}=\left\{a_{k}\right\}_{k \geq 1}$ and $\mathbf{b}=\left\{b_{k}\right\}_{k \geq 1}$ satisfy

$$
0<a_{1} \leq a_{2} \leq \cdots \leq a_{k} \leq \ldots, \quad \text { and } \quad b_{*}:=\inf _{k \geq 1} b_{k}>0 .
$$

The weighted anisotropic Sobolev space $W_{2}^{\mathbf{a}, \mathbf{b}}\left([0,1]^{d}\right)$ is defined by

$$
W_{2}^{\mathbf{a}, \mathbf{b}}\left([0,1]^{d}\right)=\left\{f \in L_{2}\left([0,1]^{d}\right): D_{j}^{b_{j}} f \in L_{2}\left([0,1]^{d}\right), \quad j=1,2, \ldots, d\right\},
$$

with norm

$$
\|f\|_{W_{2}^{\mathbf{a}, \mathbf{b}}}=\left(\|f\|_{2}^{2}+\sum_{j=1}^{d} \frac{a_{j}}{(2 \pi)^{2 b_{j}}}\left\|D_{j}^{b_{j}} f\right\|_{2}^{2}\right)^{1 / 2} .
$$

Clearly, $W_{2}^{\mathbf{a}, \mathbf{b}}\left([0,1]^{d}\right)$ is a Hilbert space. We remark that $\mathbf{b}$ is a smoothness parameter sequence, $\mathbf{a}$ is a (regulated) scaling parameter sequence with respect to the sequence $\mathbf{b}$.

It follows from (2.1) that

$$
\|f\|_{W_{2}^{\mathbf{a}, \mathbf{b}}}=\left(\sum_{\mathbf{k} \in \mathbb{Z}^{d}}\left(1+\sum_{j=1}^{d} a_{j}\left|k_{j}\right|^{2 b_{j}}\right)|\hat{f}(\mathbf{k})|^{2}\right)^{\frac{1}{2}} .
$$


If $a_{j}=(2 \pi)^{2 b_{j}}, j \in \mathbb{N}$, then $W_{2}^{\mathbf{a}, \mathbf{b}}\left([0,1]^{d}\right)$ recedes to the usual anisotropic Sobolev spaces $W_{2}^{\mathbf{b}}\left([0,1]^{d}\right)$ on the torus $[0,1]^{d}$. We emphasize that the anisotropic Sobolev spaces given in [1] are defined on the torus $\mathbb{T}^{d}=[0,2 \pi]^{d}$, not on $[0,1]^{d}$. It is easily seen that

$$
\|f\|_{W_{2}^{\mathbf{b}}}=\left(\|f\|_{2}^{2}+\sum_{j=1}^{d}\left\|D_{j}^{b_{j}} f\right\|_{2}^{2}\right)^{1 / 2}=\left(\sum_{\mathbf{k} \in \mathbb{Z}^{d}}\left(1+\sum_{j=1}^{d}\left|2 \pi k_{j}\right|^{2 b_{j}}\right)|\hat{f}(\mathbf{k})|^{2}\right)^{\frac{1}{2}}
$$

We emphasize that if we denote $\tilde{\mathbf{b}}=\left\{\tilde{b}_{j}\right\}, \tilde{b}_{j}=(2 \pi)^{2 b_{j}}, j \in \mathbb{N}$, then

$$
W_{2}^{\mathbf{b}}\left([0,1]^{d}\right)=W_{2}^{\tilde{\mathbf{b}}, \mathbf{b}}\left([0,1]^{d}\right)
$$

\subsection{Analytic Korobov spaces.}

Let $\mathbf{a}=\left\{\mathbf{a}_{j}\right\}_{j \geq 1}$ and $\mathbf{b}=\left\{\mathbf{b}_{j}\right\}_{j \geq 1}$ be the sequences satisfying (2.2). Fix $\omega \in$ $(0,1)$. We define the analytic Korobov kernel $K_{d, \mathbf{a}, 2 \mathbf{b}}$ by

$$
K_{d, \mathbf{a}, 2 \mathbf{b}}(\mathbf{x}, \mathbf{y})=\sum_{\mathbf{k} \in \mathbb{Z}^{d}} \omega_{\mathbf{k}} e^{2 \pi i \mathbf{k}(\mathbf{x}-\mathbf{y})}, \text { for all } \mathbf{x}, \mathbf{y} \in[0,1]^{d},
$$

where

$$
\omega_{\mathbf{k}}=\omega^{\sum_{j=1}^{d} a_{j}\left|k_{j}\right|^{2 b_{j}}}, \text { for all } \mathbf{k} \in \mathbb{Z}^{d} .
$$

Denote by $H\left(K_{d, \mathbf{a}, 2 \mathbf{b}}\right)$ the analytic Korobov space which is a reproducing kernel Hilbert space with the reproducing kernel $K_{d, \mathbf{a}, 2 \mathbf{b}}$. The inner product of the space $H\left(K_{d, \mathbf{a}, 2 \mathbf{b}}\right)$ is given by

$$
\langle f, g\rangle_{H\left(K_{d, \mathbf{a}, 2 \mathbf{b}}\right)}=\sum_{\mathbf{k} \in \mathbb{Z}^{d}} \hat{f}(\mathbf{k}) \overline{\hat{g}(\mathbf{k})} \omega_{\mathbf{k}}^{-1}, \quad f, g \in H\left(K_{d, \mathbf{a}, 2 \mathbf{b}}\right),
$$

where $\hat{f}(\mathbf{k}), \hat{g}(\mathbf{k}), \mathbf{k} \in \mathbb{Z}^{d}$ are the Fourier coefficients of the functions $f$ and $g$. The norm of a function $f$ in $H\left(K_{d, \mathbf{a}, 2 \mathbf{b}}\right)$ is given by

$$
\|f\|_{H\left(K_{d, \mathbf{a}, 2 \mathbf{b}}\right)}=\left(\sum_{\mathbf{k} \in \mathbb{Z}^{d}}|\hat{f}(\mathbf{k})|^{2} \omega_{\mathbf{k}}^{-1}\right)^{\frac{1}{2}}<\infty .
$$

Obviously, $\left\{e_{\mathbf{k}}\right\}_{\mathbf{k} \in \mathbb{Z}^{d}}$ is an orthonormal basis for $H\left(K_{d, \mathbf{a}, 2 \mathbf{b}}\right)$ with

$$
e_{\mathbf{k}}(\mathbf{x})=e^{2 \pi i \mathbf{k} \mathbf{x}} \omega_{\mathbf{k}}^{\frac{1}{2}}
$$

\subsection{Approximation numbers.}

Let $H$ and $G$ be two Hilbert spaces and $T$ be a compact linear operator from $H$ to $G$. The basic properties of approximation numbers $a_{n}(T: H \rightarrow G)$ are well known, see e.g., Pietsch [18, Chapter 11] and [19, Chapter 2], König [9, Section 1.b], Pinkus [20, Theorem IV.2.2], and Novak and Woźniakowski [14, Corollary 4.12].

Let $H$ be a separable Hilbert space, $\left\{e_{k}\right\}_{k=1}^{\infty}$ an orthonormal basis in $H$, and $\tau=\left\{\tau_{k}\right\}_{k=1}^{\infty}$ a sequence of positive numbers with

$$
\tau_{1} \geq \tau_{2} \geq \cdots \geq \tau_{k} \geq \cdots>0 .
$$

Let $H^{\tau}$ be a Hilbert space defined by

$$
H^{\tau}=\left\{x \in H \quad:\|x\|_{H^{\tau}}=\left(\sum_{k=1}^{\infty} \frac{\left|\left(x, e_{k}\right)\right|^{2}}{\tau_{k}^{2}}\right)^{1 / 2}<\infty\right\}
$$

According to [20, Corollary 2.6] we have the following lemma. 
Lemma 2.1. Let $H, \tau$ and $H^{\tau}$ be defined as above. Then

$$
a_{n}\left(I_{d}: H^{\tau} \rightarrow H\right)=\tau_{n}, \quad n \in \mathbb{N} .
$$

Let $\left\{W_{\mathbf{a}, \mathbf{b}, d}^{*}(l)\right\}_{l=1}^{\infty}$ be the non-increasing rearrangement of

$$
\left\{\left(1+\sum_{j=1}^{d} a_{j}\left|k_{j}\right|^{2 b_{j}}\right)^{-\frac{1}{2}}\right\}_{\mathbf{k}=\left(k_{1}, \ldots, k_{d}\right) \in \mathbb{Z}^{d}},
$$

and let $\left\{\lambda_{d, k}\right\}_{k=1}^{\infty}$ be the non-increasing rearrangement of

$$
\left\{\omega_{\mathbf{k}}\right\}_{\mathbf{k} \in \mathbb{Z}^{d}}=\left\{\omega^{\sum_{j=1}^{d} a_{j}\left|k_{j}\right|^{2 b_{j}}}\right\}_{\mathbf{k}=\left(k_{1}, \ldots, k_{d}\right) \in \mathbb{Z}^{d}},
$$

with fixed $\omega \in(0,1)$. According to Lemma 2.1, we get

$$
a_{n}\left(I_{d}: W_{2}^{\mathbf{a}, \mathbf{b}}\left([0,1]^{d}\right) \rightarrow L_{2}\left([0,1]^{d}\right)\right)=W_{\mathbf{a}, \mathbf{b}, d}^{*}(n),
$$

and

$$
a_{n}\left(\mathrm{APP}_{d}: H\left(K_{d, \mathbf{a}, 2 \mathbf{b}}\right) \rightarrow L_{2}\left([0,1]^{d}\right)\right)=\lambda_{d, n}^{1 / 2} .
$$

\subsection{General notations of tractability.}

Let $H_{d}$ and $G_{d}$ be two sequences of Hilbert space and for each $d \in \mathbb{N}, F_{d}$ be the unit ball of $H_{d}$. Assume a sequence of bounded linear operators (solution operators)

$$
S_{d}: H_{d} \rightarrow G_{d}
$$

for all $d \in \mathbb{N}$. For $n \in \mathbb{N}$ and $f \in F_{d}, S_{d} f$ can be approximated by algorithms

$$
A_{n, d}(f)=\phi_{n, d}\left(L_{1}(f), L_{2}(f), \ldots, L_{n}(f)\right),
$$

where $L_{j}, j=1,2, \ldots, n$ are continuous linear functionals on $F_{d}$ which are called general information, and $\phi_{n, d}: \mathbb{R}^{n} \rightarrow G_{d}$ is an arbitrary mapping. The worst case error $e\left(A_{n, d}\right)$ of the algorithm $A_{n, d}$ is defined as

$$
e\left(A_{n, d}\right)=\sup _{f \in F_{d}}\left\|S_{d}(f)-A_{n, d}(f)\right\|_{G_{d}} .
$$

Furthermore, we defined the $n$th minimal worst-case error as

$$
e\left(n, S_{d}\right)=\inf _{A_{n, d}} e\left(A_{n, d}\right),
$$

where the infimum is taken over all algorithms using $\mathrm{n}$ information operators $L_{1}, L_{2}, \ldots, L_{n}$. For $n=0$, we use $A_{0, d}=0$. The error of $A_{0, d}$ is called the initial error and is given by

$$
e\left(0, S_{d}\right)=e\left(A_{0, d}\right)=\sup _{f \in F_{d}}\left\|S_{d}(f)\right\|_{G_{d}} .
$$

From [14, p. 118], we know that the $n$th minimal worst-case error $e\left(n, S_{d}\right)$ with respect to arbitrary algorithms and general information in the Hilbert setting is just the $(n+1)$-approximation number $a_{n+1}\left(S_{d}: H_{d} \rightarrow G_{d}\right)$, i.e.,

$$
e\left(n, S_{d}\right)=a_{n+1}\left(S_{d}: H_{d} \rightarrow G_{d}\right) .
$$

In this paper, we consider the embedding operators $S_{d}=I_{d}$ and $S_{d}=A P P_{d}$ which are defined by (1.1) and (1.2). We note that

$$
e\left(0, I_{d}\right)=\left\|I_{d}\right\|=1 \quad \text { and } \quad e\left(0, \mathrm{APP}_{d}\right)=\left\|A P P_{d}\right\|=1 .
$$


In both cases, $e\left(0, S_{d}\right)=1$. In other words, the normalized error criterion and the absolute error criterion coincide for the approximation problems $I=\left\{I_{d}\right\}$ and $\mathrm{APP}=\left\{\mathrm{APP}_{d}\right\}$

For $\varepsilon \in(0,1)$ and $d \in \mathbb{N}$, let $n\left(\varepsilon, S_{d}\right)$ be the information complexity defined by

$$
n\left(\varepsilon, S_{d}\right)=\min \left\{n: e\left(n, S_{d}\right) \leq \varepsilon\right\},
$$

where

$$
\begin{gathered}
e\left(n, I_{d}\right)=a_{n+1}\left(I_{d}: W_{2}^{\mathbf{a}, \mathbf{b}}\left([0,1]^{d}\right) \rightarrow L_{2}\left([0,1]^{d}\right)\right), \\
e\left(n, \mathrm{APP}_{d}\right)=a_{n+1}\left(\mathrm{APP}_{d}: H\left(K_{d, \mathbf{a}, 2 \mathbf{b}}\right) \rightarrow L_{2}\left([0,1]^{d}\right)\right) .
\end{gathered}
$$

Now, we list the concepts of tractability below. We say that the approximation problem $S=\left\{S_{d}\right\}_{d \in \mathbb{N}}$ is

- strongly polynomially tractable (SPT) iff there exist non-negative numbers $C$ and $p$ such that for all $d \in \mathbb{N}, \varepsilon \in(0,1)$,

$$
n\left(\varepsilon, S_{d}\right) \leq C\left(\varepsilon^{-1}\right)^{p}
$$

- polynomially tractable (PT) iff there exist non-negative numbers $C, p$ and $q$ such that for all $d \in \mathbb{N}, \varepsilon \in(0,1)$,

$$
n\left(\varepsilon, S_{d}\right) \leq C d^{q}\left(\varepsilon^{-1}\right)^{p}
$$

- quasi-polynomially tractable $(Q P T)$ iff there exist two constants $C, t>0$ such that for all $d \in \mathbb{N}, \varepsilon \in(0,1)$,

$$
n\left(\varepsilon, S_{d}\right) \leq C \exp \left[t\left(1+\ln \varepsilon^{-1}\right)(1+\ln d)\right] ;
$$

- uniformly weakly tractable (UWT) iff for all $\alpha, \beta>0$,

$$
\lim _{\varepsilon^{-1}+d \rightarrow \infty} \frac{\ln n\left(\varepsilon, S_{d}\right)}{\left(\varepsilon^{-1}\right)^{\alpha}+d^{\beta}}=0 ;
$$

- weakly tractable (WT) iff

$$
\lim _{\varepsilon^{-1}+d \rightarrow \infty} \frac{\ln n\left(\varepsilon, S_{d}\right)}{\varepsilon^{-1}+d}=0 ;
$$

- $(s, t)$-weakly tractable $((s, t)-W T)$ for fixed positive numbers $s$ and $t$ iff

$$
\lim _{\varepsilon^{-1}+d \rightarrow \infty} \frac{\ln n\left(\varepsilon, S_{d}\right)}{\left(\varepsilon^{-1}\right)^{s}+d^{t}}=0 .
$$

In the above definitions of SPT, PT, QPT, UWT, WT, and $(s, t)-\mathrm{WT}$, if we replace $\frac{1}{\varepsilon}$ by $\left(1+\ln \frac{1}{\varepsilon}\right)$, we get the definitions of exponential convergence-strong polynomial tractability $(E C-S P T)$, exponential convergence-polynomial tractability (EC$P T)$, exponential convergence-quasi-polynomial tractability (EC-QPT), exponential convergence-uniform weak tractability (EC-UWT), exponential convergence-weak tractability $(E C-W T)$, and exponential convergence- $(s, t)$-weak tractability $(E C-(s, t)$ $W T$ ), respectively. We now give the above notions of EC-tractability in detail.

We say that $S=\left\{S_{d}\right\}_{d \in \mathbb{N}}$ is

- Exponential convergence-strong polynomial tractable (EC-SPT) iff there exist non-negative numbers $C$ and $p$ such that for all $d \in \mathbb{N}, \varepsilon \in(0,1)$,

$$
n\left(\varepsilon, S_{d}\right) \leq C\left(1+\ln \varepsilon^{-1}\right)^{p}
$$

- Exponential convergence-polynomial tractable (EC-PT) iff there exist nonnegative numbers $C, p$ and $q$ such that for all $d \in \mathbb{N}, \varepsilon \in(0,1)$,

$$
n\left(\varepsilon, S_{d}\right) \leq C d^{q}\left(1+\ln \varepsilon^{-1}\right)^{p}
$$


- Exponential convergence-quasi-polynomial tractable (EC-QPT) iff there exist two constants $C, t>0$ such that for all $d \in \mathbb{N}, \varepsilon \in(0,1)$,

$$
n\left(\varepsilon, S_{d}\right) \leq C \exp \left\{t\left[1+\ln \left(1+\ln \varepsilon^{-1}\right)\right](1+\ln d)\right\} ;
$$

- Exponential convergence-uniformly weakly tractable (EC-UWT) iff for all $\alpha, \beta>$ 0 ,

$$
\lim _{\varepsilon^{-1}+d \rightarrow \infty} \frac{\ln n\left(\varepsilon, S_{d}\right)}{\left(1+\ln \varepsilon^{-1}\right)^{\alpha}+d^{\beta}}=0
$$

- Exponential convergence-weakly tractable $(E C-W T)$ iff

$$
\lim _{\varepsilon^{-1}+d \rightarrow \infty} \frac{\ln n\left(\varepsilon, S_{d}\right)}{\left(1+\ln \varepsilon^{-1}\right)+d}=0
$$

- Exponential convergence-(s,t)-weakly tractable $(E C-(s, t)$-WT) for fixed positive $s$ and $t$ iff

$$
\lim _{\varepsilon^{-1}+d \rightarrow \infty} \frac{\ln n\left(\varepsilon, S_{d}\right)}{\left(1+\ln \varepsilon^{-1}\right)^{s}+d^{t}}=0 .
$$

\subsection{Main results.}

Let $\mathbf{a}=\left\{a_{k}\right\}_{k \geq 1}$ and $\mathbf{b}=\left\{b_{k}\right\}_{k \geq 1}$ be two sequences of positive numbers. For $t>0$ and $d \in \mathbb{N}$, denote by

$$
B_{\mathbf{a}, \mathbf{b}}^{d}(t)=\left\{x \in \mathbb{R}^{d}: \sum_{j=1}^{d} \alpha_{j}\left|x_{j}\right|^{b_{j}} \leq t\right\}
$$

the generalized ellipsoid in $\mathbb{R}^{d}$. We write $B_{\mathbf{a}, \mathbf{b}}^{d}$ instead of $B_{\mathbf{a}, \mathbf{b}}^{d}(1)$ for brevity. Clearly, when $a_{1}=a_{2}=\cdots=a_{d}=1, B_{\mathbf{a}, \mathbf{b}}^{d}$ recedes to the generalized unit ball

$$
B_{\mathbf{b}}^{d}=\left\{x \in \mathbb{R}^{d}: \sum_{j=1}^{d}\left|x_{j}\right|^{b_{j}} \leq 1\right\} .
$$

We shall show that the volume of the generalized ellipsoid $B_{\mathbf{a}, \mathbf{b}}^{d}$ is

$$
\begin{aligned}
\operatorname{vol}\left(B_{\mathbf{a}, \mathbf{b}}^{d}\right) & =2^{d} a_{1}^{-\frac{1}{b_{1}}} a_{2}^{-\frac{1}{b_{2}}} \cdots a_{d}^{-\frac{1}{b_{d}}} \frac{\Gamma\left(1+\frac{1}{b_{1}}\right) \Gamma\left(1+\frac{1}{b_{2}}\right) \cdots \Gamma\left(1+\frac{1}{b_{d}}\right)}{\Gamma\left(1+\frac{1}{b_{1}}+\frac{1}{b_{2}}+\cdots+\frac{1}{b_{d}}\right)} \\
& =a_{1}^{-\frac{1}{b_{1}}} a_{2}^{-\frac{1}{b_{2}}} \cdots a_{d}^{-\frac{1}{b_{d}}} \operatorname{vol}\left(B_{\mathbf{b}}^{d}\right),
\end{aligned}
$$

where $\Gamma(x)=\int_{0}^{\infty} t^{x-1} e^{-t} d t$ is the Gamma function. The volume of $B_{\mathbf{b}}^{d}$ is known (see [23]).

The authors in 1 investigated, among others, strong equivalence of approximation numbers $a_{n}\left(I_{d}: W_{2}^{\mathbf{R}}\left(\mathbb{T}^{d}\right) \rightarrow L_{2}\left(\mathbb{T}^{d}\right)\right)$, where $\mathbb{T}^{d}=[0,2 \pi]^{d}, \mathbf{R}=\left(R_{1}, R_{2}, \ldots, R_{d}\right) \in$ $\mathbb{R}_{+}^{d}$, and $I_{d}$ is the identity (embedding) operator. They obtained (see 1 , Theorem 2.4])

$$
\lim _{n \rightarrow \infty} n^{g(\mathbf{R})} a_{n}\left(I_{d}: W_{2}^{\mathbf{R}}\left(\mathbb{T}^{d}\right) \rightarrow L_{2}\left(\mathbb{T}^{d}\right)\right)=\left(\operatorname{vol}\left(B_{2 \mathbf{R}}^{d}\right)\right)^{g(\mathbf{R})},
$$

where $g(\mathbf{R})=\frac{1}{\frac{1}{R_{1}}+\frac{1}{R_{2}}+\cdots+\frac{1}{R_{d}}}$.

In this paper, we generalize the above result to the weighted anisotropic spaces $W_{2}^{\mathbf{a}, \mathbf{b}}\left([0,1]^{d}\right)$. We use the volume argument to obtain the asymptotic behavior of $a_{n}\left(I_{d}: W_{2}^{\mathbf{a}, \mathbf{b}}\left([0,1]^{d}\right) \rightarrow L_{2}\left([0,1]^{d}\right)\right)$. Our result can be formulated as follows. 
Theorem 2.2. Let $\mathbf{a}=\left\{a_{j}\right\}_{j \geq 1}$ and $\mathbf{b}=\left\{b_{j}\right\}_{j \geq 1}$ be two sequences of positive numbers. Then we have

$$
\lim _{n \rightarrow \infty} n^{g_{d}(\mathbf{b})} a_{n}\left(I_{d}: W_{2}^{\mathbf{a}, \mathbf{b}}\left([0,1]^{d}\right) \rightarrow L_{2}\left([0,1]^{d}\right)\right)=\left(\operatorname{vol}\left(B_{\mathbf{a}, 2 \mathbf{b}}^{d}\right)\right)^{g_{d}(\mathbf{b})},
$$

where $g_{d}(\mathbf{b})=\frac{1}{\frac{1}{b_{1}}+\frac{1}{b_{2}}+\cdots+\frac{1}{b_{d}}}$.

In particular, we have the following corollary.

Corollary 2.3. Let $\mathbf{b}=\left\{\mathbf{b}_{j}\right\}_{j \geq 1}$ be a sequences of positive numbers. Then

$$
\lim _{n \rightarrow \infty} n^{g_{d}(\mathbf{b})} a_{n}\left(I_{d}: W_{2}^{\mathbf{b}}\left([0,1]^{d}\right) \rightarrow L_{2}\left([0,1]^{d}\right)\right)=\left((2 \pi)^{-2 d} \operatorname{vol}\left(B_{2 \mathbf{b}}^{d}\right)\right)^{g_{d}(\mathbf{b})} .
$$

Remark 2.4. Let $\mathbf{a}=\left\{a_{j}\right\}_{j \geq 1}$ and $\mathbf{b}=\left\{b_{j}\right\}_{j \geq 1}$ be two sequences of positive numbers, and $g_{d}(\mathbf{b})=\frac{1}{1 / b_{1}+\cdots+1 / b_{d}}$. Theorem 2.2 indicates that the exact decay rate in $n$ of the approximation numbers $a_{n}\left(I_{d}: W_{2}^{\mathbf{a}, \mathbf{b}}\left([0,1]^{d}\right) \rightarrow L_{2}\left([0,1]^{d}\right)\right)$ is $n^{-g_{d}(\mathbf{b})}$ which is independent of $\mathbf{a}$, and the sharp constant is $\left(\operatorname{vol}\left(B_{\mathbf{a}, 2 \mathbf{b}}^{d}\right)\right)^{g_{d}(\mathbf{b})}$. We can rephrase (2.9) as strong equivalences

$$
a_{n}\left(I_{d}: W_{2}^{\mathbf{a}, \mathbf{b}}\left([0,1]^{d}\right) \rightarrow L_{2}\left([0,1]^{d}\right)\right) \sim n^{-g_{d}(\mathbf{b})}\left(\operatorname{vol}\left(B_{\mathbf{a}, 2 \mathbf{b}}^{d}\right)\right)^{g_{d}(\mathbf{b})} .
$$

The novelty of Theorem 2.2 is that they give strong equivalences and provide asymptotically optimal (sharp) constants, for arbitrary fixed $d$, $\mathbf{a}$, and $\mathbf{b}$.

Remark 2.5. Comparing (2.8) with (2.10), we get that the sharp constants of the approximation numbers of anisotropic Sobolev embeddings on the torus $[a, b]^{d}$ depend on the volume of the torus. We can show that

$$
\lim _{n \rightarrow \infty} n^{g_{d}(\mathbf{b})} a_{n}\left(I_{d}: W_{2}^{\mathbf{b}}\left([a, b]^{d}\right) \rightarrow L_{2}\left([a, b]^{d}\right)\right)=\left(\left(\frac{b-a}{2 \pi}\right)^{2 d} \operatorname{vol}\left(B_{2 \mathbf{b}}^{d}\right)\right)^{g_{d}(\mathbf{b})} .
$$

Remark 2.6. According to [3, Theorem 3.1], we know that the condition $g_{d}(\mathbf{b})>\frac{1}{2}$ is a sufficient and necessary condition for the embedding from $W_{2}^{\mathbf{a}, \mathbf{b}}\left([0,1]^{d}\right)$ into $L_{\infty}\left([0,1]^{d}\right)$ or $C\left([0,1]^{d}\right)$. Furthermore, for $g_{d}(\mathbf{b})>\frac{1}{2}$, using the proof technique of 3. Theorem 4.3] we can show

$\lim _{n \rightarrow \infty} n^{g_{d}(\mathbf{b})-\frac{1}{2}} a_{n}\left(I_{d}: W_{2}^{\mathbf{a}, \mathbf{b}}\left([0,1]^{d}\right) \rightarrow L_{\infty}\left([0,1]^{d}\right)\right)=\left(2 g_{d}(\mathbf{b})-1\right)^{-\frac{1}{2}}\left(\operatorname{vol}\left(B_{\mathbf{a}, 2 \mathbf{b}}^{d}\right)\right)^{g_{d}(\mathbf{b})}$.

Note that the above equality also holds if we replace $L_{\infty}\left([0,1]^{d}\right)$ by $C\left([0,1]^{d}\right)$.

Next, we establish a relationship of the information complexities $n\left(\varepsilon, I_{d}\right)$ and $n\left(\varepsilon, \mathrm{APP}_{d}\right)$. Such relationship is crucial for obtaining sufficient and necessary conditions of various notions of tractability of the approximation problem $I=\left\{I_{d}\right\}$.

Theorem 2.7. For $\varepsilon \in(0,1), d \in \mathbb{N}$, we have

$$
n\left(\varepsilon, \operatorname{APP}_{d}\right)=n\left(\left(\frac{\ln \varepsilon^{-2}}{\ln \omega^{-1}}+1\right)^{-\frac{1}{2}}, I_{d}\right)
$$

and

$$
n\left(\varepsilon, I_{d}\right)=n\left(\omega^{\frac{\varepsilon^{-2}-1}{2}}, \mathrm{APP}_{d}\right),
$$

where $n\left(\varepsilon, S_{d}\right)$ is given in (2.7), $S_{d}=I_{d}$ or $\mathrm{APP}_{d}$. 
We know that the classical tractability of the multivariate problem APP has been solved completely in [8, 13, 6]. For the EC-tractability of APP, the sufficient and necessary conditions for EC-SPT, EC-PT, EC-QPT, EC-UWT, EC-WT, and EC- $(s, t)$-WT with $\max (s, t)>1$ were given in [6], and for EC- $(s, t)$-WT with $\max (s, t) \leq 1$ and $\min (s, t)<1$ in 22 . Based on Theorem 2.7 we obtain the following tractability results of the approximation problem $I=\left\{I_{d}\right\}$.

Theorem 2.8. Consider the approximation problem $I=\left\{I_{d}\right\}$ in the worst case setting with the sequences $\mathbf{a}$ and $\mathbf{b}$ satisfying (2.2). Then

(i) SPT holds iff PT holds iff

$$
\sum_{j=1}^{\infty} b_{j}^{-1}<\infty \quad \text { and } \quad \underline{\lim }_{j \rightarrow \infty} \frac{\ln a_{j}}{j}>0
$$

(ii) QPT holds iff

$$
\sup _{d \in \mathbb{N}} \frac{\sum_{j=1}^{d} b_{j}^{-1}}{1+\ln d}<\infty \quad \text { and } \quad \varliminf_{j \rightarrow \infty} \frac{(1+\ln j) \ln a_{j}}{j}>0 ;
$$

(iii) UWT holds iff

$$
\lim _{j \rightarrow \infty} \frac{\ln a_{j}}{\ln j}=\infty
$$

(iv) WT holds iff

$$
\lim _{j \rightarrow \infty} \frac{a_{j}}{j}=\infty ;
$$

(v) $(s, t)-W T$ with $\max (s / 2, t)>1$ always holds;

(vi) $(2,1)-W T$ holds iff

$$
\lim _{j \rightarrow \infty} a_{j}=\infty
$$

(vii) $(2, t)-W T$ with $t<1$ holds iff

$$
\lim _{j \rightarrow \infty} \frac{a_{j}}{\ln j}=\infty
$$

(viii) $(s, t)-W T$ with $s<2$ and $t \leq 1$ holds iff

$$
\lim _{j \rightarrow \infty} \frac{a_{j}}{j^{(2-s) / s}}=\infty \text {. }
$$

In particular, let $\mathbf{b}=\left\{b_{j}\right\}$ satisfy

$$
0<b_{1} \leq b_{2} \leq \cdots \leq b_{j} \leq \ldots,
$$

and $\tilde{\mathbf{b}}=\left\{\tilde{b}_{j}\right\}, \tilde{b}_{j}=(2 \pi)^{2 b_{j}}, j \in \mathbb{N}$. Then $W_{2}^{\mathbf{b}}\left([0,1]^{d}\right)=W_{2}^{\tilde{\mathbf{b}}, \mathbf{b}}\left([0,1]^{d}\right)$. By Theorem 2.8 we have the following result.

Theorem 2.9. Consider the approximation problem $\tilde{I}=\left\{\tilde{I}_{d}\right\}_{d \in \mathbb{N}}$ in the worst case setting with the sequence b satisfying (2.13), where

$$
\tilde{I}_{d}: W_{2}^{\mathbf{b}}\left([0,1]^{d}\right) \rightarrow L_{2}\left([0,1]^{d}\right) \text { with } \quad \tilde{I}_{d}(f)=f .
$$

Then we have

(i) $\tilde{I}$ is SPT iff $\tilde{I}$ is PT iff

$$
\sum_{j=1}^{\infty} b_{j}^{-1}<\infty
$$


(ii) $\tilde{I}$ is QPT iff

$$
\sup _{d \in \mathbb{N}} \frac{\sum_{j=1}^{d} b_{j}^{-1}}{1+\ln d}<\infty
$$

(iii) UWT holds iff

$$
\lim _{j \rightarrow \infty} \frac{b_{j}}{\ln j}=\infty
$$

(iv) WT holds iff

$$
\lim _{j \rightarrow \infty} \frac{(2 \pi)^{2 b_{j}}}{j}=\infty
$$

(v) $(s, t)-W T$ with $\max (s / 2, t)>1$ always holds;

(vi) $(2,1)$-WT holds iff

$$
\lim _{j \rightarrow \infty} b_{j}=\infty
$$

(vii) $(2, t)-$ WT with $t<1$ holds iff

$$
\lim _{j \rightarrow \infty} \frac{(2 \pi)^{2 b_{j}}}{\ln j}=\infty
$$

(viii) $(s, t)$-WT with $s<2$ and $t \leq 1$ holds iff

$$
\lim _{j \rightarrow \infty} \frac{(2 \pi)^{2 b_{j}}}{j^{(2-s) / s}}=\infty .
$$

\section{Strong Equivalences of approximation numbers}

This section is devoted to studying strong equivalence of the approximation numbers $a_{n}\left(I_{d}: W_{2}^{\mathbf{a}, \mathbf{b}}\left([0,1]^{d}\right) \rightarrow L_{2}\left([0,1]^{d}\right)\right)$, where $\mathbf{a}=\left\{a_{j}\right\}_{j \geq 1}$ and $\mathbf{b}=\left\{b_{j}\right\}_{j \geq 1}$ are two sequences of positive numbers. In this section we do not need to assume that $\mathbf{a}, \mathbf{b}$ satisfy (2.2).

We know from [23] that the volume of the generalized unit ball $B_{\mathbf{b}}^{d}$ is

$$
\operatorname{vol}\left(B_{\mathbf{b}}^{d}\right)=\operatorname{vol}\left\{x \in \mathbb{R}^{d}: \sum_{j=1}^{d}\left|x_{j}\right|^{b_{j}} \leq 1\right\}=2^{d} \frac{\Gamma\left(1+\frac{1}{b_{1}}\right) \Gamma\left(1+\frac{1}{b_{2}}\right) \cdots \Gamma\left(1+\frac{1}{b_{d}}\right)}{\Gamma\left(1+\frac{1}{b_{1}}+\frac{1}{b_{2}}+\cdots+\frac{1}{b_{d}}\right)} .
$$

Lemma 3.1. Let $\mathbf{a}=\left\{a_{j}\right\}_{j \geq 1}$ and $\mathbf{b}=\left\{b_{j}\right\}_{j \geq 1}$ be two sequences of positive numbers. Then

$$
\operatorname{vol}\left(B_{\mathbf{a}, \mathbf{b}}^{d}\right)=a_{1}^{-\frac{1}{b_{1}}} a_{2}^{-\frac{1}{b_{2}}} \cdots a_{d}^{-\frac{1}{b_{d}}} \operatorname{vol}\left(B_{\mathbf{b}}^{d}\right) .
$$

Proof. We make a change of variables

$$
y_{1}=x_{1} a_{1}^{\frac{1}{b_{1}}}, \quad y_{2}=x_{2} a_{2}^{\frac{1}{b_{2}}}, \quad \cdots, \quad y_{d}=x_{d} a_{d}^{\frac{1}{b_{d}}}
$$

that deforms $B_{\mathbf{a}, \mathbf{b}}^{d}$ into $B_{\mathbf{b}}^{d}$. The Jacobian determinant is $J(y)=a_{1}^{-\frac{1}{b_{1}}} a_{2}^{-\frac{1}{b_{2}}} \cdots a_{d}^{-\frac{1}{b_{d}}}$. By the change of variables formula, we get

$$
\operatorname{vol}\left(B_{\mathbf{a}, \mathbf{b}}^{d}\right)=\int_{B_{\mathbf{a}, \mathbf{b}}^{d}} 1 d x=\int_{B_{\mathbf{b}}^{d}} J(y) d y=a_{1}^{-\frac{1}{b_{1}}} a_{2}^{-\frac{1}{b_{2}}} \cdots a_{d}^{-\frac{1}{b_{d}}} \operatorname{vol}\left(B_{\mathbf{b}}^{d}\right) .
$$

The proof of Lemma 3.1 is finished. 
Lemma 3.2. Let $\mathbf{a}=\left\{a_{j}\right\}_{j \geq 1}$ and $\mathbf{b}=\left\{b_{j}\right\}_{j \geq 1}$ be two sequences of positive numbers. Then

$$
\operatorname{vol}\left(B_{\mathbf{a}, \mathbf{b}}^{d}(t)\right)=t^{\frac{1}{b_{1}}+\frac{1}{b_{2}}+\cdots+\frac{1}{b_{d}}} \operatorname{vol}\left(B_{\mathbf{a}, \mathbf{b}}^{d}\right)=t^{\frac{1}{g_{d}^{(\mathbf{b})}}} \operatorname{vol}\left(B_{\mathbf{a}, \mathbf{b}}^{d}\right),
$$

where $g_{d}(\mathbf{b})=\frac{1}{\frac{1}{b_{1}}+\frac{1}{b_{2}}+\cdots+\frac{1}{b_{d}}}$.

Proof. We make a change of variables

$$
y_{1}=x_{1} t^{-\frac{1}{b_{1}}}, y_{2}=x_{2} t^{-\frac{1}{b_{2}}}, \ldots, y_{d}=x_{d} t^{-\frac{1}{b_{d}}}
$$

that deforms $B_{\mathbf{a}, \mathbf{b}}^{d}(t)$ into $B_{\mathbf{a}, \mathbf{b}}^{d}$. The Jacobian determinant is $J(y)=t^{\frac{1}{b_{1}}+\frac{1}{b_{2}}+\ldots+\frac{1}{b_{d}}}$. By the change of variables formula, we obtain

$$
\operatorname{vol}\left(B_{\mathbf{a}, \mathbf{b}}^{d}(t)\right)=\int_{B_{\mathbf{a}, \mathbf{b}}^{d}(t)} 1 d x=\int_{B_{\mathbf{a}, \mathbf{b}}^{d}} J(y) d y=t^{\frac{1}{b_{1}}+\frac{1}{b_{2}}+\cdots+\frac{1}{b_{d}}} \operatorname{vol}\left(B_{\mathbf{a}, \mathbf{b}}^{d}\right) .
$$

The proof of Lemma 3.2 is finished.

Lemma 3.3. Let $\mathbf{a}=\left\{a_{j}\right\}_{j \geq 1}$ and $\mathbf{b}=\left\{b_{j}\right\}_{j \geq 1}$ be two sequences of positive numbers, and let $p_{d}=\max \left\{1,2 b_{1}, 2 b_{2}, \ldots, 2 b_{d}\right\}$. Then for any $\mathbf{x}, \mathbf{y} \in \mathbb{R}^{d}$, we have

$$
\left(\sum_{j=1}^{d} a_{j}\left|x_{j}+y_{j}\right|^{2 b_{j}}\right)^{\frac{1}{p_{d}}} \leq\left(\sum_{j=1}^{d} a_{j}\left|x_{j}\right|^{2 b_{j}}\right)^{\frac{1}{p_{d}}}+\left(\sum_{j=1}^{d} a_{j}\left|y_{j}\right|^{2 b_{j}}\right)^{\frac{1}{p_{d}}} .
$$

Proof. It follows from [1, Lemma 3.2] that for $\mathbf{x}=\left(x_{1}, \ldots, x_{d}\right), \mathbf{y}=\left(y_{1}, \ldots, y_{d}\right), \mathbf{x}, \mathbf{y} \in$ $\mathbb{R}^{d}$, we have

$$
\left(\sum_{j=1}^{d}\left|x_{j}+y_{j}\right|^{2 b_{j}}\right)^{1 / p_{d}} \leq\left(\sum_{j=1}^{d}\left|x_{j}\right|^{2 b_{j}}\right)^{1 / p_{d}}+\left(\sum_{j=1}^{d}\left|y_{j}\right|^{2 b_{j}}\right)^{1 / p_{d}} .
$$

If we replace $x_{j}, y_{j}, j=1,2, \ldots, d$ by $a_{j}^{\frac{1}{2 b_{j}}} x_{j}, a_{j}^{\frac{1}{2 b_{j}}} y_{j}, j=1,2, \ldots, d$ in the above inequality, then we get

$$
\left(\sum_{j=1}^{d}\left|a_{j}^{\frac{1}{2 b_{j}}} x_{j}+a_{j}^{\frac{1}{2 b_{j}}} y_{j}\right|^{2 b_{j}}\right)^{1 / p_{d}} \leq\left(\sum_{j=1}^{d}\left|a_{j}^{\frac{1}{2 b_{j}}} x_{j}\right|^{2 b_{j}}\right)^{1 / p_{d}}+\left(\sum_{j=1}^{d}\left|a_{j}^{\frac{1}{2 b_{j}}} y_{j}\right|^{2 b_{j}}\right)^{1 / p_{d}}
$$

which gives (3.3). Lemma 3.3 is proved.

Proof of Theorem 2.2.

It follows from (2.5) that

$$
a_{n}\left(I_{d}: W_{2}^{\mathbf{a}, \mathbf{b}}\left([0,1]^{d}\right) \rightarrow L_{2}\left([0,1]^{d}\right)\right)=W_{\mathbf{a}, \mathbf{b}, d}^{*}(n),
$$

where $\left\{W_{\mathbf{a}, \mathbf{b}, d}^{*}(l)\right\}_{l=1}^{\infty}$ is the non-increasing rearrangement of

$$
\left\{\left(1+\sum_{j=1}^{d} a_{j}\left|k_{j}\right|^{2 b_{j}}\right)^{-\frac{1}{2}}\right\}_{\mathbf{k}=\left(k_{1}, \ldots, k_{d}\right) \in \mathbb{Z}^{d}}
$$

For $m \in \mathbb{N}$, let $C(m, \mathbf{a}, \mathbf{b}, d)$ denote the cardinality of the set

$$
\left\{\mathbf{k}: \sum_{j=1}^{d} a_{j}\left|k_{j}\right|^{2 b_{j}} \leq m^{p_{d}}, \mathbf{k} \in \mathbb{Z}^{d}\right\}
$$


where $p_{d}=\max \left\{1,2 b_{1}, 2 b_{2}, \ldots, 2 b_{d}\right\}$. It follows that for $n>C(m, \mathbf{a}, \mathbf{b}, d)$,

$$
a_{n}\left(I_{d}: W_{2}^{\mathbf{a}, \mathbf{b}}\left([0,1]^{d}\right) \rightarrow L_{2}\left([0,1]^{d}\right)\right) \leq\left(1+m^{p_{d}}\right)^{-\frac{1}{2}} .
$$

and for $n \leq C(m, \mathbf{a}, \mathbf{b}, d)$,

$$
a_{n}\left(I_{d}: W_{2}^{\mathbf{a}, \mathbf{b}}\left([0,1]^{d}\right) \rightarrow L_{2}\left([0,1]^{d}\right)\right) \geq\left(1+m^{p_{d}}\right)^{-\frac{1}{2}} .
$$

For any $m \in \mathbb{N}$, let $Q_{k}$ be a cube with center $\mathbf{k}$, sides parallel to the axes and side-length 1 . For

$$
\mathbf{x} \in \bigcup_{\substack{\mathbf{k} \in \mathbb{Z}^{d} \\ \sum_{j=1}^{d} a_{j}\left|k_{j}\right|^{2 b_{j}} \leq m^{p_{d}}}} Q_{\mathbf{k}}
$$

there exists a $\mathbf{k} \in \mathbb{Z}^{d}$ such that $\sum_{j=1}^{d} a_{j}\left|k_{j}\right|^{2 b_{j}} \leq m^{p_{d}}$ and $\mathbf{x} \in Q_{\mathbf{k}}$. It follows from the definition of $Q_{\mathbf{k}}$ that

$$
\left|x_{j}-k_{j}\right| \leq \frac{1}{2}, \quad j=1,2, \ldots, d .
$$

By (3.3) we have

$$
\begin{aligned}
\left(\sum_{j=1}^{d} a_{j}\left|x_{j}\right|^{2 b_{j}}\right)^{\frac{1}{p_{d}}} & \leq\left(\sum_{j=1}^{d} a_{j}\left|x_{j}-k_{j}\right|^{2 b_{j}}\right)^{\frac{1}{p_{d}}}+\left(\sum_{j=1}^{d} a_{j}\left|k_{j}\right|^{2 b_{j}}\right)^{\frac{1}{p_{d}}} \\
& \leq\left(\sum_{j=1}^{d} a_{j} 2^{-2 b_{j}}\right)^{\frac{1}{p_{d}}}+m .
\end{aligned}
$$

It follows that

$$
\bigcup_{\substack{\mathbf{k} \in \mathbb{Z}^{d} \\ \sum_{j=1}^{d} a_{j}\left|k_{j}\right|^{2 b_{j}} \leq m^{p_{d}}}} Q_{\mathbf{k}} \subset B_{\mathbf{a}, 2 \mathbf{b}}^{d}\left(\left(m+\left(\sum_{j=1}^{d} a_{j} 2^{-2 b_{j}}\right)^{\frac{1}{p_{d}}}\right)^{p_{d}}\right) .
$$

Using the same technique, we get

$$
B_{\mathbf{a}, 2 \mathbf{b}}^{d}\left(\left(m-\left(\sum_{j=1}^{d} a_{j} 2^{-2 b_{j}}\right)^{\frac{1}{p_{d}}}\right)_{+}^{p_{d}}\right) \subset \bigcup_{\substack{\mathbf{k} \in \mathbb{Z}^{d} \\ \sum_{j=1}^{d} a_{j}\left|k_{j}\right|^{2 b_{j}} \leq m^{p_{d}}}} Q_{\mathbf{k}},
$$

where $a_{+}$is equal to $a$ if $a>0$ and 0 if $a<0$. We note that the volume of the set

$$
\operatorname{vol}\left(\bigcup_{\substack{\mathbf{k} \in \mathbb{Z}^{d} \\ \sum_{j=1}^{d} a_{j}\left|k_{j}\right|^{2 b_{j}} \leq m^{p_{d}}}} Q_{\mathbf{k}}\right)=\#\left\{\mathbf{k}: \sum_{j=1}^{d} a_{j}\left|k_{j}\right|^{2 b_{j}} \leq m^{p_{d}}, \mathbf{k} \in \mathbb{Z}^{d}\right\}
$$

is just $C(m, \mathbf{a}, \mathbf{b}, d)$, where \#A denotes the number of elements in a set $A$. We set

$$
C_{\mathbf{a}, \mathbf{b}, d}=\left(\sum_{j=1}^{d} a_{j} 2^{-2 b_{j}}\right)^{\frac{1}{p_{d}}}
$$

By (3.5), (3.4), and (3.2) we get

$$
\left(m-C_{\mathbf{a}, \mathbf{b}, d}\right)_{+}^{\frac{p_{d}}{g_{d}(2 \mathbf{b})}} \operatorname{vol}\left(B_{\mathbf{a}, 2 \mathbf{b}}^{d}\right) \leq C(m, \mathbf{a}, \mathbf{b}, d) \leq\left(m+C_{\mathbf{a}, 2 \mathbf{b}, d}\right)^{\frac{p_{d}}{g_{d}^{(2 \mathbf{b})}}},
$$


where $g_{d}(2 \mathbf{b})=2 g_{d}(\mathbf{b})=\frac{2}{\frac{1}{b_{1}}+\frac{1}{b_{2}}+\cdots+\frac{1}{b_{d}}}$. We write

$$
a_{n}\left(I_{d}\right)=a_{n}\left(I_{d}: W_{2}^{\mathbf{a}, \mathbf{b}}\left([0,1]^{d}\right) \rightarrow L_{2}\left([0,1]^{d}\right)\right)
$$

We also write

$$
A(m, \mathbf{a}, \mathbf{b}, d)=\left(m+C_{\mathbf{a}, \mathbf{b}, d}\right)^{\frac{p_{d}}{2 g_{d}(\mathbf{b})}} \operatorname{vol}\left(B_{\mathbf{a}, 2 \mathbf{b}}^{d}\right)
$$

and

$$
B(m, \mathbf{a}, \mathbf{b}, d)=\left(m-C_{\mathbf{a}, \mathbf{b}, d}\right)_{+}^{\frac{p_{d}}{2 g_{d}(\mathbf{b})}} \operatorname{vol}\left(B_{\mathbf{a}, 2 \mathbf{b}}^{d}\right) .
$$

On the one hand, we suppose that $A(m, \mathbf{a}, \mathbf{b}, d)<n \leq A(m+1, \mathbf{a}, \mathbf{b}, d)$. By (3.6) we get

$$
n>C(m, \mathbf{a}, \mathbf{b}, d),
$$

which implies

$$
a_{n}\left(I_{d}\right) \leq\left(1+m^{p_{d}}\right)^{-\frac{1}{2}} .
$$

It follows that

$$
\begin{aligned}
n^{g_{d}(\mathbf{b})} a_{n}\left(I_{d}\right) & \leq \frac{(A(m+1, \mathbf{a}, \mathbf{b}, d))^{g_{d}(\mathbf{b})}}{\left(1+m^{p_{d}}\right)^{\frac{1}{2}}} \\
& \leq \frac{\left(m+1+C_{\mathbf{a}, \mathbf{b}, d}\right)^{\frac{p_{d}}{2}}\left(\operatorname{vol}\left(B_{\mathbf{a}, 2 \mathbf{b}}^{d}\right)\right)^{g_{d}(\mathbf{b})}}{\left(1+m^{p_{d}}\right)^{\frac{1}{2}}}
\end{aligned}
$$

Obviously, we have

$$
\lim _{m \rightarrow \infty} \frac{\left(m+1+C_{\mathbf{a}, \mathbf{b}, d}\right)^{\frac{p_{d}}{2}}}{\left(1+m^{p_{d}}\right)^{\frac{1}{2}}}=1
$$

which implies that

$$
\lim _{n \rightarrow \infty} n^{g_{d}(\mathbf{b})} a_{n}\left(I_{d}\right) \leq\left(\operatorname{vol}\left(B_{\mathbf{a}, 2 \mathbf{b}}^{d}\right)\right)^{g_{d}(\mathbf{b})} .
$$

On the other hand, we suppose that $B(m, \mathbf{a}, \mathbf{b}, d) \leq n<B(m+1, \mathbf{a}, \mathbf{b}, d)$ as $n$ is sufficiently large. By (3.6) we get

$$
n<C(m+1, \mathbf{a}, \mathbf{b}, d)
$$

which implies

$$
a_{n}\left(I_{d}\right) \geq\left(1+(m+1)^{p_{d}}\right)^{-\frac{1}{2}}
$$

It follows that

$$
\begin{aligned}
n^{g_{d}(\mathbf{b})} a_{n}\left(I_{d}\right) & \geq \frac{(B(m, \mathbf{a}, \mathbf{b}, d))^{g_{d}(\mathbf{b})}}{\left(1+(m+1)^{p_{d}}\right)^{\frac{1}{2}}} \\
& \geq \frac{\left(m-C_{\mathbf{a}, \mathbf{b}, d}\right)^{\frac{p_{d}}{2}}}{\left(1+(m+1)^{p_{d}}\right)^{\frac{1}{2}}}\left(\operatorname{vol}\left(B_{\mathbf{a}, 2 \mathbf{b}}^{d}\right)\right)^{g_{d}(\mathbf{b})}
\end{aligned}
$$

We have

which implies that

$$
\lim _{m \rightarrow \infty} \frac{\left(m-C_{\mathbf{a}, \mathbf{b}, d}\right)^{\frac{p_{d}}{2}}}{\left(1+(m+1)^{p_{d}}\right)^{\frac{1}{2}}}=1,
$$

$$
\lim _{n \rightarrow \infty} n^{g_{d}(\mathbf{b})} a_{n}\left(I_{d}\right) \geq\left(\operatorname{vol}\left(B_{\mathbf{a}, 2 \mathbf{b}}^{d}\right)\right)^{g_{d}(\mathbf{b})} .
$$

Combining (3.7) with (3.8), we obtain (2.9).

Theorem 2.2 is proved. 


\section{Tractability Results of Weighted Anisotropic EmbedDings}

In this section, we first give the proof of Theorem 2.7. Next we establish the relationship between tractability of $I=\left\{I_{d}\right\}_{d \in \mathbb{N}}$ and EC-tractability of APP $=$ $\left\{\mathrm{APP}_{d}\right\}_{d \in \mathbb{N}}$ based on Theorem 2.7, where $I_{d}$ and $\mathrm{APP}_{d}$ are given by (1.1) and (1.2). Finally we show Theorems 2.8 and 2.9 .

Proof of Theorem 2.7.

For any $\varepsilon \in(0,1), \quad d \in \mathbb{N}$, by (2.7), (2.5), and (2.6) we have

$$
\begin{aligned}
n\left(\varepsilon, I_{d}\right) & =\min \left\{n: a_{n+1}\left(I_{d}: W_{2}^{\mathbf{a}, \mathbf{b}} \rightarrow L_{2}\left([0,1]^{d}\right)\right) \leq \varepsilon\right\} \\
& =\min \left\{n: W_{\mathbf{a}, \mathbf{b}, d}^{*}(n+1) \leq \varepsilon\right\}
\end{aligned}
$$

and

$$
\begin{aligned}
n\left(\varepsilon, \mathrm{APP}_{d}\right) & =\min \left\{n: a_{n+1}\left(\mathrm{APP}_{d}: H\left(K_{d, \mathbf{a}, 2 \mathbf{b}}\right) \rightarrow L_{2}\left([0,1]^{d}\right)\right) \leq \varepsilon\right\} \\
& =\min \left\{n: \lambda_{d, n+1} \leq \varepsilon^{2}\right\}
\end{aligned}
$$

where $\left\{W_{\mathbf{a}, \mathbf{b}, d}^{*}(l)\right\}_{l=1}^{\infty}$ is the non-increasing rearrangement of

$$
\left\{\left(1+\sum_{j=1}^{d} a_{j}\left|k_{j}\right|^{2 b_{j}}\right)^{-\frac{1}{2}}\right\}_{\mathbf{k}=\left(k_{1}, \ldots, k_{d}\right) \in \mathbb{Z}^{d}}
$$

and $\left\{\lambda_{d, k}\right\}_{k=1}^{\infty}$ is the non-increasing rearrangement of

$$
\left\{\omega_{\mathbf{k}}\right\}_{\mathbf{k} \in \mathbb{Z}^{d}}=\left\{\omega^{\sum_{j=1}^{d} a_{j}\left|k_{j}\right|^{2 b_{j}}}\right\}_{\mathbf{k}=\left(k_{1}, \ldots, k_{d}\right) \in \mathbb{Z}^{d}}
$$

with the fixed $\omega \in(0,1)$.

For any $\varepsilon_{1} \in(0,1)$, by (4.2) we have

$$
\begin{aligned}
n\left(\varepsilon_{1}, \mathrm{APP}_{d}\right) & =\min \left\{n: \lambda_{d, n+1} \leq \varepsilon_{1}^{2}\right\}=\#\left\{j \in \mathbb{N}: \lambda_{d, j}>\varepsilon_{1}^{2}\right\} \\
& =\#\left\{\mathbf{k} \in \mathbb{Z}^{d}: \omega^{\sum_{j=1}^{d} a_{j}\left|k_{j}\right|^{2 b_{j}}}>\varepsilon_{1}^{2}\right\} \\
& =\#\left\{\mathbf{k} \in \mathbb{Z}^{d}: \omega^{-\sum_{j=1}^{d} a_{j}\left|k_{j}\right|^{2 b_{j}}}<\varepsilon_{1}^{-2}\right\} \\
& =\#\left\{\mathbf{k} \in \mathbb{Z}^{d}: \sum_{j=1}^{d} a_{j}\left|k_{j}\right|^{2 b_{j}}<\frac{\ln \varepsilon_{1}^{-2}}{\ln \omega^{-1}}\right\} .
\end{aligned}
$$

We set

$$
\varepsilon_{2}=\left(\frac{\ln \varepsilon_{1}^{-2}}{\ln \omega^{-1}}+1\right)^{-\frac{1}{2}}
$$

Then $\varepsilon_{2} \in(0,1)$ and

$$
\varepsilon_{2}^{-2}-1=\frac{\ln \varepsilon_{1}^{-2}}{\ln \omega^{-1}}
$$


By (4.1) we obtain

$$
\begin{aligned}
n\left(\varepsilon_{2}, I_{d}\right) & =\min \left\{n: W_{\mathbf{a}, \mathbf{b}, d}^{*}(n+1) \leq \varepsilon_{2}\right\}=\#\left\{l \in \mathbb{N}: W_{\mathbf{a}, \mathbf{b}, d}^{*}(l)>\varepsilon_{2}\right\} \\
& =\#\left\{\mathbf{k} \in \mathbb{Z}^{d}:\left(1+\sum_{j=1}^{d} a_{j}\left|k_{j}\right|^{2 b_{j}}\right)^{-\frac{1}{2}}>\varepsilon_{2}\right\} \\
& =\#\left\{\mathbf{k} \in \mathbb{Z}^{d}: \sum_{j=1}^{d} a_{j}\left|k_{j}\right|^{2 b_{j}}<\varepsilon_{2}^{-2}-1\right\} \\
& =\#\left\{\mathbf{k} \in \mathbb{Z}^{d}: \sum_{j=1}^{d} a_{j}\left|k_{j}\right|^{2 b_{j}}<\frac{\ln \varepsilon_{1}^{-2}}{\ln \omega^{-1}}\right\} .
\end{aligned}
$$

It follows that

$$
n\left(\varepsilon_{1}, \mathrm{APP}_{d}\right)=n\left(\varepsilon_{2}, I_{d}\right)=n\left(\left(\frac{\ln \varepsilon_{1}^{-2}}{\ln \omega^{-1}}+1\right)^{-\frac{1}{2}}, I_{d}\right),
$$

which gives (2.11). Using the same method we can prove (2.12).

Theorem 2.7 is proved.

Theorem 4.1. Consider the approximation problems $I=\left\{I_{d}\right\}$ and $\mathrm{APP}=\left\{\mathrm{APP}_{d}\right\}$ in the worst case setting, where $I_{d}$ and $\mathrm{APP}_{d}$ are given by (1.1) and (1.2). We have

(i) for fixed $s, t>0, I$ is $(2 s, t)-W T$ iff $\mathrm{APP}$ is $E C$ - $(s, t)-W T$;

(ii) $I$ is $U W T$ iff APP is EC-UWT;

(iii) $I$ is $Q P T$ iff APP is EC-QPT;

(iv) $I$ is PT iff APP is EC-PT;

(v) $I$ is SPT iff APP is EC-SPT.

Proof. (i) First we prove the sufficiency. Assume that $I$ is $(2 s, t)$-WT. For $\varepsilon \in(0,1)$, we set

$$
\varepsilon_{1}=\left(\frac{\ln \varepsilon^{-2}}{\ln \omega^{-1}}+1\right)^{-\frac{1}{2}} \in(0,1)
$$

Then we have

$$
d+\varepsilon^{-1} \rightarrow \infty \text { iff } d+\varepsilon_{1}^{-1} \rightarrow \infty .
$$

By the definition of $(s, t)$-WT we have

$$
\lim _{d+\varepsilon_{1}^{-1} \rightarrow \infty} \frac{\ln n\left(\varepsilon_{1}, I_{d}\right)}{d^{t}+\left(\varepsilon_{1}^{-1}\right)^{2 s}}=0 .
$$

It follows from (2.11) that

$$
\frac{\ln n\left(\varepsilon, \mathrm{APP}_{d}\right)}{d^{t}+\left(1+\ln \varepsilon^{-1}\right)^{s}}=\frac{\ln n\left(\varepsilon_{1}, I_{d}\right)}{d^{t}+\varepsilon_{1}^{-2 s}} \frac{d^{t}+\left(\frac{\ln \varepsilon^{-2}}{\ln \omega^{-1}}+1\right)^{s}}{d^{t}+\left(1+\ln \varepsilon^{-1}\right)^{s}} .
$$

Noting that

$$
\varepsilon_{1}^{-2}=\frac{\ln \varepsilon^{-2}}{\ln \omega^{-1}}+1 \leq C_{1}\left(1+\ln \varepsilon^{-1}\right),
$$

where $C_{1}=\max \left\{1, \frac{2}{\ln \omega^{-1}}\right\}$, we get

$$
\frac{d^{t}+\left(\frac{\ln \varepsilon^{-2}}{\ln \omega^{-1}}+1\right)^{s}}{d^{t}+\left(1+\ln \varepsilon^{-1}\right)^{s}} \leq \frac{d^{t}+C_{1}^{s}\left(1+\ln \varepsilon^{-1}\right)^{s}}{d^{t}+\left(1+\ln \varepsilon^{-1}\right)^{s}} \leq C_{1}^{s} .
$$


It follows that

$$
\lim _{d+\varepsilon^{-1} \rightarrow \infty} \frac{\ln n\left(\varepsilon, \mathrm{APP}_{d}\right)}{d^{t}+\left(1+\ln \varepsilon^{-1}\right)^{s}}=0,
$$

which yields that APP is EC- $(s, t)-\mathrm{WT}$.

Next we show the necessity. Suppose that APP is EC- $(s, t)$-WT. For $\varepsilon \in(0,1)$, we set

$$
\varepsilon_{2}=\omega^{\frac{\varepsilon^{-2}-1}{2}} \in(0,1) .
$$

Then we have

$$
d+\varepsilon^{-1} \rightarrow \infty \text { iff } d+\varepsilon_{2}^{-1} \rightarrow \infty .
$$

By the definition of EC- $(s, t)-\mathrm{WT}$ we have

$$
\lim _{d+\varepsilon_{2}^{-1} \rightarrow \infty} \frac{\ln n\left(\varepsilon_{2}, \mathrm{APP}_{d}\right)}{d^{t}+\left(1+\ln \varepsilon_{2}^{-1}\right)^{s}}=0 .
$$

By (2.12), we have

$$
\frac{\ln n\left(\varepsilon, I_{d}\right)}{d^{t}+\left(\varepsilon^{-1}\right)^{2 s}}=\frac{\ln n\left(\varepsilon_{2}, \mathrm{APP}_{d}\right)}{d^{t}+\left(1+\ln \varepsilon_{2}^{-1}\right)^{s}} \frac{d^{t}+\left(1+\frac{\varepsilon^{-2}-1}{2} \ln \omega^{-1}\right)^{s}}{d^{t}+\left(\varepsilon^{-1}\right)^{2 s}} .
$$

Noting that

$$
1+\ln \varepsilon_{2}^{-1}=1+\frac{\varepsilon^{-2}-1}{2} \ln \omega^{-1} \leq 1+\varepsilon^{-2} \ln \omega^{-1} \leq C_{2} \varepsilon^{-2}
$$

where $C_{2}=1+\ln \omega^{-1}$, we obtain

$$
\frac{d^{t}+\left(1+\frac{\varepsilon^{-2}-1}{2} \ln \omega^{-1}\right)^{s}}{d^{t}+\left(\varepsilon^{-1}\right)^{2 s}} \leq \frac{d^{t}+C_{2}^{s} \varepsilon^{-2 s}}{d^{t}+\varepsilon^{-2 s}} \leq C_{2}^{s} .
$$

It follows that

$$
\lim _{d+\varepsilon^{-1} \rightarrow \infty} \frac{\ln n\left(\varepsilon, I_{d}\right)}{d^{t}+\left(\varepsilon^{-1}\right)^{2 s}}=0
$$

which leads to that $I$ is $(2 s, t)$-WT. Hence $(i)$ is proved.

(ii) We note that $I$ is UWT iff for all $\alpha, \beta>0, I$ is $(\alpha, \beta)$-WT. By (i) we obtain that $I$ is UWT iff for all $\alpha, \beta>0$, APP is EC- $\left(\frac{\alpha}{2}, \beta\right)-\mathrm{WT}$. This is equivalent to that APP is EC-UWT. Hence (ii) is proved.

(iii) We first assume that $I$ is QPT. Then there exist two constants $C, t>0$ such that for all $d \in \mathbb{N}, \varepsilon \in(0,1)$,

$$
n\left(\varepsilon, I_{d}\right) \leq C \exp \left[t\left(1+\ln \varepsilon^{-1}\right)(1+\ln d)\right] .
$$

By Theorem 2.7 and (4.4) we have

$$
\begin{aligned}
n\left(\varepsilon, \mathrm{APP}_{d}\right) & =n\left(\varepsilon_{1}, I_{d}\right) \leq C \exp \left[t\left(1+\ln \varepsilon_{1}^{-1}\right)(1+\ln d)\right] \\
& \leq C \exp \left\{t\left[1+\frac{1}{2} \ln \left(C_{1}\left(1+\ln \varepsilon^{-1}\right)\right)\right](1+\ln d)\right\} \\
& \leq C \exp \left\{t_{1}\left[1+\ln \left(1+\ln \varepsilon^{-1}\right)\right](1+\ln d)\right\},
\end{aligned}
$$

where $\varepsilon_{1}$ is given by (4.3), $t_{1}=t\left(1+\frac{\ln C_{1}}{2}\right), C_{1}=\max \left\{1, \frac{2}{\ln \omega^{-1}}\right\}$. This means that $\mathrm{APP}$ is EC-QPT.

Next, we assume that APP is EC-QPT. We want to show that $I$ is QPT. There exist two constants $C, t>0$ such that for all $d \in \mathbb{N}, \varepsilon \in(0,1)$,

$$
n\left(\varepsilon, \mathrm{APP}_{d}\right) \leq C \exp \left\{t\left[1+\ln \left(1+\ln \varepsilon^{-1}\right)\right](1+\ln d)\right\} .
$$


According to Therorem 2.7 and (4.6), we obtain

$$
\begin{aligned}
n\left(\varepsilon, I_{d}\right) & =n\left(\varepsilon_{2}, \mathrm{APP}_{d}\right) \leq C \exp \left\{t\left[1+\ln \left(1+\ln \varepsilon_{2}^{-1}\right)\right](1+\ln d)\right\} \\
& \leq C \exp \left\{t\left[1+\ln \left(C_{2} \varepsilon^{-2}\right)\right](1+\ln d)\right\} \\
& \left.\leq C \exp \left[t_{2}\left(1+\ln \varepsilon^{-1}\right)\right)(1+\ln d)\right],
\end{aligned}
$$

where $\varepsilon_{2}$ is given by (4.5), $t_{2}=t \max \left\{1+\ln C_{2}, 2\right\}, C_{2}=1+\ln \omega^{-1}$. This means that $I$ is QPT. Hence (iii) is proved.

(iv) First we assume that $I$ is PT. There exist non-negative numbers $C, p$ and $q$ such that for all $d \in \mathbb{N}, \varepsilon \in(0,1)$,

$$
n\left(\varepsilon, I_{d}\right) \leq C d^{q} \varepsilon^{-p} .
$$

By Theorem 2.7, (4.3), (4.4) we have

$$
n\left(\varepsilon, \mathrm{APP}_{d}\right)=n\left(\varepsilon_{1}, I_{d}\right) \leq C d^{q} \varepsilon_{1}^{-p} \leq C C_{1}^{p / 2} d^{q}\left(1+\ln \varepsilon^{-1}\right)^{p / 2},
$$

which means that APP is EC-PT.

Next, suppose that APP is EC-PT. There exist non-negative numbers $C, p$ and $q$ such that for all $d \in \mathbb{N}, \varepsilon \in(0,1)$,

$$
n\left(\varepsilon, \mathrm{APP}_{d}\right) \leq C d^{q}\left(1+\ln \varepsilon^{-1}\right)^{p} .
$$

By Theorem 2.7, (4.5), (4.6) we have

$$
n\left(\varepsilon, I_{d}\right)=n\left(\varepsilon_{2}, \mathrm{APP}_{d}\right) \leq C d^{q}\left(1+\ln \varepsilon_{2}^{-1}\right)^{p} \leq C C_{2}^{p} d^{q} \varepsilon^{-2 p},
$$

which implies that $I$ is PT. Hence $(i v)$ is proved.

$(v)$ The proof is the same as the one of $(i v)$.

The proof of Theorem 4.1 is finished.

Proof of Theorem 2.8.

According to [6] and [22], we have the following EC-tractability results of APP:

- EC-SPT holds iff EC-PT holds iff

$$
\sum_{j=1}^{\infty} b_{j}^{-1}<\infty \quad \text { and } \quad \varliminf_{j \rightarrow \infty} \frac{\ln a_{j}}{j}>0 .
$$

- EC-QPT holds iff

$$
\sup _{d \in \mathbb{N}} \frac{\sum_{j=1}^{d} b_{j}^{-1}}{1+\ln d}<\infty \quad \text { and } \quad \varliminf_{j \rightarrow \infty} \frac{(1+\ln j) \ln a_{j}}{j}>0 .
$$

- EC-UWT holds iff

$$
\lim _{j \rightarrow \infty} \frac{\ln a_{j}}{\ln j}=\infty .
$$

- EC- $(s, t)$-WT with $\max (s, t)>1$ always holds.

- EC-WT holds iff

$$
\lim _{j \rightarrow \infty} a_{j}=\infty .
$$

- EC-(1,t)-WT with $t<1$ holds iff

$$
\lim _{j \rightarrow \infty} \frac{a_{j}}{\ln j}=\infty .
$$


- EC- $(s, t)$-WT with $s<1$ and $t \leq 1$ holds iff

$$
\lim _{j \rightarrow \infty} \frac{a_{j}}{j^{(1-s) / s}}=\infty \text {. }
$$

Hence, Theorem 2.8 follows from Theorem 4.1 and the above results immediately.

Proof of Theorem 2.9.

We note that if $\tilde{\mathbf{b}}=\left\{\tilde{b}_{j}\right\}, \tilde{b}_{j}=(2 \pi)^{2 b_{j}}, j \in \mathbb{N}$, then

$$
W_{2}^{\mathbf{b}}\left([0,1]^{d}\right)=W_{2}^{\tilde{\mathbf{b}}, \mathbf{b}}\left([0,1]^{d}\right) .
$$

It follows from Theorem 2.8 that

(1) $\tilde{I}$ is $\mathrm{SPT}$ iff $\tilde{I}$ is PT iff

$$
\sum_{j=1}^{\infty} b_{j}^{-1}<\infty \quad \text { and } \quad \underline{\lim _{j \rightarrow \infty}} \frac{b_{j}}{j}>0
$$

(2) $\tilde{I}$ is QPT iff

$$
\sup _{d \in \mathbb{N}} \frac{\sum_{j=1}^{d} b_{j}^{-1}}{1+\ln d}<\infty \quad \text { and } \quad \varliminf_{j \rightarrow \infty} \frac{(1+\ln j) b_{j}}{j}>0 ;
$$

(3) (iii), (iv), (v), (vi), (vii), (viii) hold.

Hence, in order to show Theorem 2.9, it suffices to prove that

(a) if $\sum_{j=1}^{\infty} b_{j}^{-1}<\infty$, then we have $\varliminf_{j \rightarrow \infty} \frac{b_{j}}{j}>0$;

(b) if $\sup _{d \in \mathbb{N}} \frac{\sum_{j=1}^{d} b_{j}^{-1}}{1+\ln d}<\infty$, then $\varliminf_{j \rightarrow \infty} \frac{(1+\ln j) b_{j}}{j}>0$.

First we prove (a). Assume that $B:=\sum_{j=1}^{\infty} b_{j}^{-1}<\infty$. We have

$$
B \geq \sum_{j=1}^{k} b_{j}^{-1} \geq k b_{k}^{-1} .
$$

It follows that

which yields that

$$
\frac{b_{k}}{k} \geq \frac{1}{B}
$$

$$
\varliminf_{k \rightarrow \infty} \frac{b_{k}}{k} \geq \frac{1}{B}>0
$$

Hence (a) holds.

Next we show (b). Assume that $D:=\sup _{d \in \mathbb{N}} \frac{\sum_{j=1}^{d} b_{j}^{-1}}{1+\ln d}<\infty$. We have

$$
D \geq \frac{\sum_{j=1}^{k} b_{j}^{-1}}{1+\ln k} \geq \frac{k b_{k}^{-1}}{1+\ln k}
$$

It follows that

$$
\frac{(1+\ln k) b_{k}}{k} \geq \frac{1}{D}
$$


which yields that

$$
\varliminf_{k \rightarrow \infty} \frac{(1+\ln k) b_{k}}{k} \geq \frac{1}{D}>0
$$

Hence (b) holds.

This completes the proof of Theorem 2.9.

Acknowledgment This work was supported by the National Natural Science Foundation of China (Project no. 11671271) and the Natural Science Foundation of Beijing Municipality (1172004).

\section{REFERENCES}

[1] J. Chen, H. Wang, Preasymptotics and asymptotics of approximation numbers of anisotropic Sobolev embeddings, J. Complexity, 39 (2017) 94-110.

[2] J. Chen, H. Wang, Approximation numbers of Sobolev and Gevrey type embeddings on the sphere and on the ball - Preasymptotics, asymptotics, and tractability, J. Complexity 50 (2019) 1-24.

[3] F. Cobos, T. Kühn, W. Sickel, Optimal approximation of multivariate periodic Sobolev functions in the sup-norm, J. Funct. Anal. 270 (11) (2016) 4196-4212.

[4] J. Dick, P. Kritzer, F. Pillichshammer, H. Woźniakowski, Approximation of analytic functions in Korobov spaces, J. Complexity 30 (2014) 2-28.

[5] J. Dick, G. Larcher, F. Pillichshammer, H. Woźniakowski, Exponential convergence and tractability of multivariate integration for Korobov spaces, Math. Comp. 80 (2011) 905-930.

[6] C. Irrgeher, P. Kritzer, F. Pillichshammer, H. Woźniakowski, Tractability of multivariate approximation defined over Hilbert spaces with exponential weights, J. Approx. Theory 207 (2016) 301-338.

[7] P. Kritzer, F. Pillichshammer, H. Woźniakowski, Multivariate integration of infinitely many times differentiable functions in weighted Korobov spaces, Math. Comp. 83 (2014) 1189-1206.

[8] P. Kritzer, F. Pillichshammer, H. Woźniakowski, Tractability of multivariate analytic problems, in: P. Kritzer, H. Niederreiter, F. Pillichshammer, A. Winterhof (Eds.), Uniform Distribution and Quasi-Monte Carlo Methods. Discrepancy, Integration and Applications, De Gruyter, Berlin, 2014, pp. 147-170.

[9] T. Kühn, A lower estimate for entropy numbers, J. Approx. Theory, 110 (2001) 120-124.

[10] T. Kühn, S. Mayer, T. Ullrich, Counting via entropy: new preasymptotics for the approximation numbers of Sobolev embeddings, SIAM J. Numer. Anal. 54 (6) (2016) 3625-3647.

[11] T. Kühn, W. Sickel, T. Ullrich, Approximation numbers of Sobolev embeddings-Sharp constants and tractability, J. Complexity 30 (2014) 95-116.

[12] T. Kühn, W. Sickel, T. Ullrich, Approximation of mixed order Sobolev functions on the $d$-torus: asymptotics, preasymptotics, and $d$-dependence, Constr. Approx. 42(3) (2015) 353398

[13] Y. Liu, G. Xu, A note on tractability of multivariate analytic problems, J. Comlexity, 34 (2016) 42-49.

[14] E. Novak, H. Woźniakowski, Tractablity of Multivariate Problems, Volume I: Linear Information, EMS, Zürich, 2008.

[15] E. Novak, H. Woźniakowski, Tractablity of Multivariate Problems, Volume II: Standard Information for Functionals, EMS, Zürich, 2010

[16] E. Novak, H. Woźniakowski, Tractablity of Multivariate Problems, Volume III: Standard Information for Operators, EMS, Zürich, 2012.

[17] A. Papageorgiou, I. Petras, A new criterion for tractability of multivariate problems, J. Complexity 30 (2014) 604-619.

[18] A. Pietsch, Operator Ideals, North-Holland, Amsterdam, 1980.

[19] A. Pietsch, Eigenvalues and s-Numbers, Cambridge University Press, Cambridge, 1987.

[20] A. Pinkus, n-Widths in Approximation Theory, Ergeb. Math. Grenzgeb., vol. 3.7, Springer, Berlin, 1985.

[21] G. Xu, Exponential convergence-tractability of general linear problems in the average case setting, J. Complexity 31 (2015) 617-636. 
[22] H. Wang, A note about EC- $(s, t)$-weak tractability of multivariate approximation with analytic Korobov kernels, to appear in J. Complexity. Also see http://arxiv.org/abs/1808.01470.

[23] X. Wang, Volumes of generalized unit balls, Math.Mag. 78 (5) (2005) 390-395.

[24] A. Werschulz, H. Woźniakowski, Tractability of multivariate approximation over weighted standard Sobolev spaces, J. Complexity, in press (2018).

School of Mathematical Sciences, BCMiIs, Capital Normal University, Beijing 100048 , China.

E-mail address: 1047695025@qq.com(D. Hao). wanghp@cnu.edu.cn(H. Wang). 